\title{
Beneficial effects of a combination of Rho-kinase inhibitor and ACE inhibitor on tubulointerstitial fibrosis induced by unilateral ureteral obstruction
}

\author{
Yosuke Takeda $^{1}$, Toshio Nishikimi ${ }^{1}$, Kazumi Akimoto ${ }^{2}$, Hiroaki Matsuoka ${ }^{1}$ and Toshihiko Ishimitsu ${ }^{1}$
}

We and others recently reported that long-term Rho-kinase inhibition has renoprotective effects. This study was designed to compare the effects of an angiotensin-converting enzyme (ACE) inhibitor (imidapril), a Rho-kinase inhibitor (fasudil) and a combination of them both on renal interstitial fibrosis induced by unilateral ureteral obstruction (UUO). We also attempted to elucidate the mechanism involved. Imidapril $\left(50 \mathrm{mgl}^{-1}\right)$, fasudil $\left(1 \mathrm{gl}^{-1}\right)$ or a combination of them both was given in drinking water to mice, and their effects were compared on renal interstitial fibrosis induced by UUO. We assessed histological findings, monocyte/macrophage infiltration, myofibroblast differentiation, oxidative stress and the expression of various mRNA in the kidney by UUO. Eleven days after UUO, wild-type kidney was characterized by increased fibrotic area, dihydroethidium (DHE)positive area, $\alpha$-smooth muscle actin (SMA)-positive area, F4/80-positive area and the increased expression of various mRNA. Fasudil and imidapril similarly improved fibrotic area $(-23 \%,-15 \%)$, DHE-positive area $(-13 \%,-11 \%), \alpha$-SMA-positive area $(-22 \%,-15 \%), \mathrm{F} 4 / 80$-positive area $(-42 \%,-34 \%)$ and the expression of various mRNA, most of which were significant $(P<0.05)$. The combination of imidapril and fasudil further improved fibrotic area $(-52 \%)$, DHE-positive area $(-26 \%), \alpha$-SMApositive area $(-33 \%), \mathrm{F} 4 / 80$-positive area $(-62 \%)$ and the expression of various mRNA (all $\boldsymbol{P}<0.05$ vs. monotherapy). Compared with either agent alone, the combination of an ACE inhibitor and a Rho-kinase inhibitor was more effective for the prevention of renal interstitial fibrosis because of the inhibition of transforming growth factor- $\beta /$ collagen, monocyte/macrophage infiltration, myofibroblast differentiation, inflammation and the oxidative stress pathway.

Hypertension Research (2010) 33, 965-973; doi:10.1038/hr.2010.112; published online 22 July 2010

Keywords: ACE inhibitor; renal fibrosis; Rho-kinase inhibitor; unilateral ureteral obstruction

\section{INTRODUCTION}

Rho, a small guanosine triphosphate-binding protein, functions as a molecular switch in various cellular functions, including formation of stress fibers, focal adhesions, regulation of calcium ion sensitivity, production of cytokines and the regulation of G1 to $S$ phase in cell cycle progression. ${ }^{1,2}$ The cellular function and signal transduction of Rho-kinase have been extensively studied. The discovery of specific inhibitors of Rho-kinase greatly advanced the knowledge of Rho/Rhokinase pathway in vitro and in vivo. ${ }^{3}$ As Rho enhances the $\mathrm{Ca}^{2+}$ sensitization of vascular smooth muscle cells by inhibiting myosin phosphatase activity, the effects of Rho/Rho-kinase on the tone of blood vessels and the function of Rho in the pathogenesis of arteriosclerosis have been intensively investigated. ${ }^{4}$

Recent studies have revealed that the Rho/Rho-kinase pathway is involved in the pathophysiology of various cardiovascular diseases, such as hypertension, angina pectoris, myocardial infarction and pulmonary hypertension. ${ }^{5}$ However, few studies have investigated the function of Rho/Rho-kinase in kidney disease. ${ }^{6}$ Earlier studies revealed that specific Rho-kinase inhibitors, such as Y-27632 or fasudil, significantly attenuate the tubulointerstitial fibrosis in the kidney induced by unilateral ureteral obstruction (UUO). ${ }^{7,8}$ More recently, we and other investigators have shown that the Rho-kinase inhibitor fasudil attenuates glomerulosclerosis in salt-induced hypertensive rats, ${ }^{9}$ subtotally nephrectomized spontaneously hypertensive rats, ${ }^{10}$ malignant hypertensive rats, ${ }^{11}$ severely hypertensive rats, ${ }^{12}$ aldosterone-induced glomerular injury in rats, ${ }^{13}$ diabetic renal injury in rats ${ }^{14}$ and $\mathrm{N}(\mathrm{G})$-nitro-L-arginine methyl ester-induced hypertensive renal injury rats. ${ }^{15}$

Angiotensin-converting enzyme (ACE) inhibitors have been shown to attenuate renal damage including glomerulosclerosis, tubulointerstitial fibrosis and arteriosclerosis and improve kidney function. ${ }^{16}$ In the clinical setting, ACE inhibitors are a first line therapy for kidney disease and are widely used for the prevention of renal disease; ${ }^{17}$ however, their effects are limited, and a new drug or combination therapy involving ACE inhibitors is desired. Therefore, in this study, we examined the effect of a combination of an ACE inhibitor and

${ }^{1}$ Department of Hypertension and Cardiorenal Medicine, Dokkyo Medical University, Mibu, Tochigi, Japan and ${ }^{2}$ Clinical Research Center, Dokkyo Medical University, Mibu, Tochigi, Japan

Correspondence: Dr T Nishikimi, Department of Medicine and Clinical Science, Kyoto University Graduate School of Medicine, 54, Shogoin-Kawara-cho, Sakyo-ku, Kyoto 606-8507, Japan. 
a Rho-kinase inhibitor on renal fibrosis induced by UUO and compared its effects with the effects of either agent alone. We also attempted to elucidate the mechanism involved.

\section{METHODS}

This study was approved by our institutional Animal Care Committee, and all of the procedures were in accordance with the National Institutes of Health Guide for the Care and Use of Laboratory Animals.

\section{Experimental animals and design}

Male C57BL/6 mice, aged 3-6 months ( $n=40$ ), were used in this study. A UUO kidney disease model was induced in the mice (20-25 g body weight (BW), 3-6 months of age) by left ureteral ligation as described earlier. ${ }^{8,18}$ Sham-operated mice (the same operation without ureter ligation) were also produced. After the UUO operation, the mice were randomly assigned to four groups: untreated $(n=8)$, imidapril treated $\left(50 \mathrm{mgl}^{-1}\right)(n=8)$, fasudil treated $\left(\mathrm{gl}^{-1}\right)(n=8)$ or a combination group $(n=8)$. Imidapril $\left(50 \mathrm{mgl}^{-1}\right)$, fasudil $\left(1 \mathrm{gl}^{-1}\right)$ or a combination of them was given in drinking water to the mice for 11 days, and their effects were compared with untreated mice. After oral administration, fasudil is metabolized to hydroxyfasudil, a major active metabolite of fasudil that specifically inhibits Rho-kinase. ${ }^{19}$ We determined the dose of fasudil and imidapril according to the methods of earlier studies, which function as a renoprotective drugs in kidney disease. ${ }^{12,16}$ Kidney tissue samples collected at day 11 after UUO were used for histology, immunohistochemistry, renal superoxide anion detections and a real-time polymerase chain reaction (PCR) analysis study.

\section{Tail-cuff blood pressure analysis}

Systolic blood pressure (BP) and pulse rate were measured noninvasively in conscious and restrained mice as described earlier, ${ }^{20}$ before and at 11 days after the operation.

\section{Assessment of renal interstitial fibrosis}

A part of obstructed kidney was excised and immersed in neutralized formalin for histological examination. The area of fibrotic lesions in the interstitium (fibrosis area) was determined on sections stained by Masson's trichrome method to stain collagen fibers (stained blue), using a computer-aided manipulator program as described earlier. ${ }^{18}$

\section{Assessment of interstitial myofibroblast differentiation}

Immunohistochemical analysis using an antibody against anti- $\alpha$-smooth muscle actin (SMA), a marker of myofibroblast expression, was performed as reported earlier. ${ }^{18}$ Quantification of the $\alpha$-SMA-positive area was determined using a computer-aided manipulator program as described earlier. ${ }^{18}$

\section{Assessment of monocyte/macrophage infiltration}

Immunohistochemical analysis using an antibody against $\mathrm{F} 4 / 80$, a monocyte/ macrophage marker, was performed as reported earlier. ${ }^{18}$ Quantification of the F4/80-positive area was determined using a computer-aided manipulator program as described earlier. ${ }^{18}$

\section{In situ detection of renal superoxide anion}

Superoxide $\left(\mathrm{O}^{2-}\right)$ anion generation in renal tissue was evaluated using the fluorescent dye dihydroethidium (DHE) as earlier reported. ${ }^{15} \mathrm{~A}$ part of renal tissue embedded in optimal cutting temperature was quickly frozen and cut with a cryostat ( $5 \mu \mathrm{m}$, cross-section). The sections were incubated with DHE $1 \mu \mathrm{moll}^{-1}$ in a light-protected humidified chamber at $37^{\circ} \mathrm{C}$ for $5 \mathrm{~min}$. After washing the sections with phosphate buffer saline, images were acquired with identical acquisition parameters using a fluorescence microscope (Olympus AX80; Olympus, Tokyo, Japan). DHE specifically reacts with intracellular $\mathrm{O}^{2-}$ and is converted to the red fluorescent compound ethidium, which then binds irreversibly to double-stranded DNA and appears as punctuate nuclear staining. Fluorescence intensity was quantified using Mac SCOPE software (Mitani Shoji, Fukui, Japan) and recorded as mean gray scale intensity. ${ }^{15}$

\section{Quantification of mRNA by real-time PCR}

All of the procedures used for mRNA extraction and cDNA synthesis were described in detail in earlier reports. ${ }^{20}$

The gene expression levels of collagen-I, collagen-III, transforming growth factor (TGF)- $\beta$, monocyte chemoattractant protein (MCP)-1, tumor necrosis factor (TNF)- $\alpha$, nicotinamide adenine dinucleotide phosphate (NADPH) subunit (p22phox, gp91 phox, p40phox, p47phox, p67phox), angiotensinogen (ANG), renin, ACE, angiotensin II type-1 receptor (AT1-R), RhoA, RhoB, RhoC and glyceroaldehyde 3-phosphate dehydrogenase (GAPDH) in the renal tissue were determined by real-time quantitative reverse-transcription polymerase chain reaction using Applied Biosystem 7700 (Applied Biosystem, Foster City, CA, USA) and specific primers as reported earlier. ${ }^{20}$

\section{Statistical analysis}

All data are expressed as mean \pm s.d. Statistical comparisons among the five groups were carried out using analysis of variance. If appropriate, the data were compared with the Bonferroni's post hoc test for multiple comparisons. $P<0.05$ was considered to indicate statistical significance.

\section{RESULTS}

BW, BP, pulse rate and kidney weight in the five groups

The physiological profiles of the five groups at day 11 after the UUO operation are shown in Table 1. There were no significant differences in BW among the five groups. There were no significant differences in normal side kidney weight/BW among the five groups, either. In disease side, creation of a UUO significantly decreased kidney weight/BW compared with sham-operated mice; however, there were no differences in kidney weight/BW among the four UUO groups. There were no significant differences in systolic BP or pulse rate before the operation among the five groups (data not shown). UUO operation did not change the systolic BP. Fasudil treatment did not change the systolic BP, either. As a result, there were no differences in systolic BP among the sham, untreated-UUO and fasudil-treated UUO groups. In contrast, imidapril significantly reduced the systolic BP. Cotreatment with imidapril and fasudil also significantly reduced BP.

Table 1 BW, SBP, heart rate and Kid W/BW in five groups at 11 days after UUO production

\begin{tabular}{|c|c|c|c|c|c|}
\hline & Sham & Untreated UUO & Fasudil-treated UUO & Imidapril-treated UUO & Imidapril+fasudil-treated UUO \\
\hline Number & 8 & 8 & 8 & 8 & 8 \\
\hline SBP (mm Hg) & $102 \pm 3$ & $102 \pm 3$ & $101 \pm 4$ & $92 \pm 5^{* \dagger \neq}$ & $95 \pm 3 * 1 \neq$ \\
\hline PR (beats per minute) & $703 \pm 30$ & $723 \pm 38$ & $713 \pm 27$ & $735 \pm 17$ & $734 \pm 21$ \\
\hline BW at 11 days & $24.6 \pm 2.4$ & $24.9 \pm 4.7$ & $24.4 \pm 5.0$ & $24.6 \pm 2.4$ & $23.3 \pm 3.1$ \\
\hline Kid W/BW $\left(\mathrm{g} \mathrm{kg}^{-1}\right)$ (normal side) & $7.12 \pm 0.32$ & $7.18 \pm 0.50$ & $7.39 \pm 1.09$ & $6.95 \pm 0.24$ & $7.27 \pm 0.51$ \\
\hline Kid W/BW $\left(\mathrm{g} \mathrm{kg}^{-1}\right)$ (disease side) & $7.16 \pm 0.22$ & $4.93 \pm 1.02 *$ & $5.01 \pm 0.53^{*}$ & $4.96 \pm 0.63^{*}$ & $5.59 \pm 1.22^{*}$ \\
\hline
\end{tabular}

Abbreviations: BW, body weight; Kid W, kidney weight; PR, pulse rate; SBP, systolic blood pressure; UUO, unilateral ureteral obstruction

${ }^{*} P<0.05$ vs. sham; ${ }^{\dagger} P<0.05$ vs. untreated UUO; ${ }^{\ddagger} P<0.05$ vs. fasudil-treated UUO 


\section{Interstitial fibrosis of the kidneys in the five groups}

The representative appearances of Masson's trichrome-stained sections 11 days after ureteral obstruction are shown in Figure 1. Interstitial fibrosis of the obstructed kidney was prominent in the untreated mice compared with that in the normal kidney of the sham-operated mice (Figures $1 \mathrm{a}$ and $\mathrm{b}$ ). Fasudil or imidapril treatment slightly ameliorated the interstitial fibrosis compared with that in the untreated mice (Figures $1 \mathrm{c}$ and $\mathrm{d}$ ). The combination of fasudil and imidapril further ameliorated renal interstitial fibrosis (Figure 1e). The tubular structure was also slightly more preserved in the fasudil- or imidapril-treated mice compared with the untreated mice and was further preserved in the mice treated with both drugs. In the measurement of the fibrous area in obstructed kidneys by computed quantitative analysis, untreated-UUO mice showed a greater fibrous area than the shamoperated mice $(P<0.01)$, and fasudil or imidapril treatment significantly reduced the fibrous area (both $P<0.05$ vs. untreated), and the combination of fasudil and imidapril further reduced the fibrous area $(P<0.05$ vs. fasudil- or imidapril-treated group) (Figure 1f).

\section{Interstitial $\alpha$-SMA expression in the kidneys in the five groups}

The induction of interstitial myofibroblasts was assessed by immunohistochemical detection of $\alpha$-SMA. Positive staining for $\alpha$-SMA was seen only in vascular smooth muscle cells, but not in the interstitial space in the normal kidney of the sham-operated mice (Figure 2a). Intense immunostaining of $\alpha$-SMA was observed in the peritubular interstitium in addition to the vascular smooth muscle cells of the arterioles in obstructed kidney in untreated mice 11 days after ureteral ligation (Figure 2b). Fasudil or imidapril treatment reduced the appearance of $\alpha$-SMA-positive myofibroblasts in the interstitium (Figures $2 \mathrm{c}$ and $\mathrm{d}$ ), and the combination of fasudil and imidapril further ameliorated it (Figure 2e). The results of the quantitative analysis of $\alpha$-SMA immunostaining are shown in Figure $2 \mathrm{f}$. The $\alpha$-SMA-positive area in obstructed kidneys was significantly greater in the untreated-UUO mice than in the sham-operated mice $(P<0.01)$, and fasudil or imidapril treatment significantly reduced the positive area (both $P<0.05 v s$. untreated), and the combination of fasudil and imidapril further reduced it $(P<0.05 v s$. fasudil- or imidapril-treated group) (Figure 2f).

\section{Interstitial F4/80 expression in the kidneys in the five groups} Monocyte/macrophage migration was assessed by immunohistochemical detection of F4/80-positive cells. F4/80-positive cells in the interstitium are shown in Figure 3. The number of F4/80-positive cells in the interstitium was obviously increased in the untreated mice compared with the normal kidneys of the sham-operated mice (Figures $3 \mathrm{a}$ and $\mathrm{b}$ ). Fasudil or imidapril treatment similarly reduced the number of F4/80-positive cells in the interstitium (Figures $3 \mathrm{c}$ and d), and the combination of fasudil and imidapril further reduced it (Figure 3e). The results of the quantitative analysis of F4/80-positive area are shown in Figure 3f. The F4/80-positive area in the obstructed kidneys was markedly greater in the untreated-UUO mice than in the sham-operated mice $(P<0.01)$, and fasudil or imidapril treatment significantly reduced the F4/80-positive area (both $P<0.05$ vs. untreated), and the combination of fasudil and imidapril further reduced it $(P<0.05$ vs. fasudil- or imidapril-treated group) (Figure 3f).

\section{DHE staining in the kidneys in the five groups}

Intracellular $\mathrm{O}_{2}^{-}$levels, a marker of oxidative stress, in the renal cortex were measured using DHE and fluorescence microscopy. Representative results of the DHE staining in the five groups are shown in Figure 4 . The fluorescence intensity in the interstitium was obviously

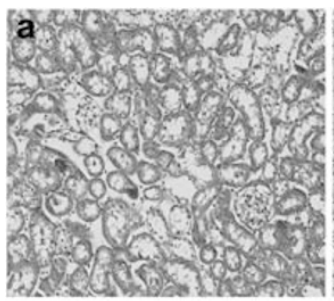

Normal

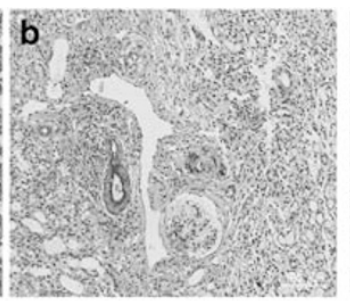

Untreated

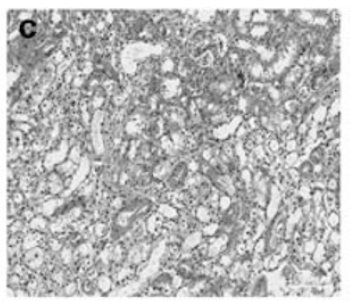

Fasudil treated

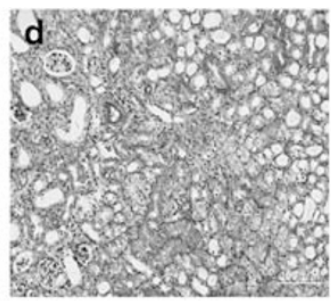

Imidapril treated

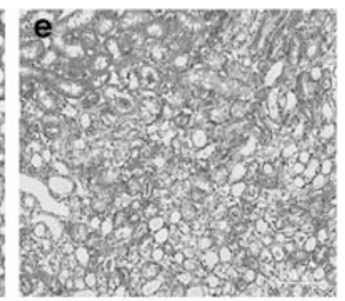

Fas+Imida treated

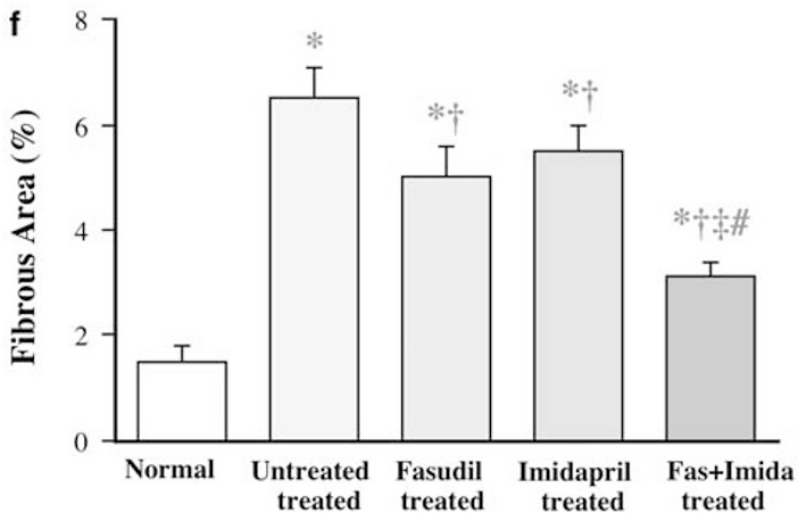

Figure 1 The morphological appearance of the interstitium as shown by Masson's trichrome staining in normal kidney in sham-operated mice (a) and in obstructed kidneys in untreated (b), fasudil-treated (c), imidapril-treated (d) and fasudil- and imidapril-treated mice (e) 11 days after the production of UUO are shown. The areas of the fibrotic lesions of the interstitium (fibrosis area) are shown in (f). Data are expressed as mean $\pm \mathrm{s}$.d. The number of mice was $6-8$ in each group. ${ }^{*} P<0.05$ vs. normal, ${ }^{\dagger} P<0.05$ vs. untreated, ${ }^{\ddagger} P<0.05$ vs. fasudil-treated, ${ }^{\#} P<0.05$ vs. imidapril-treated mice. 


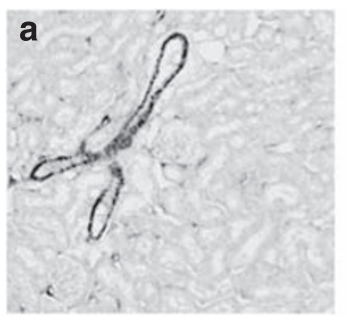

Normal

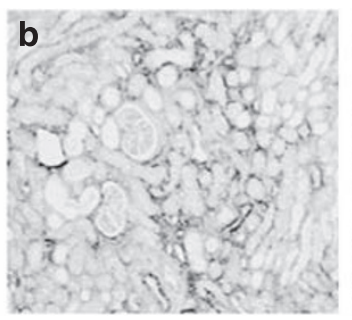

Untreated

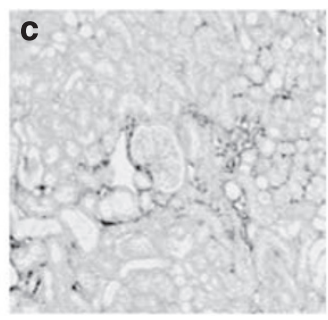

Fasudil

treated

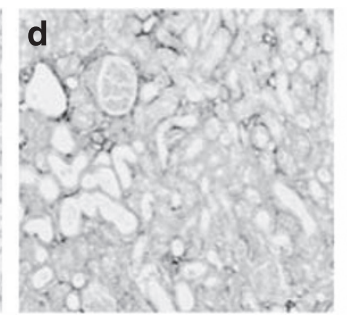

Imidapril

treated

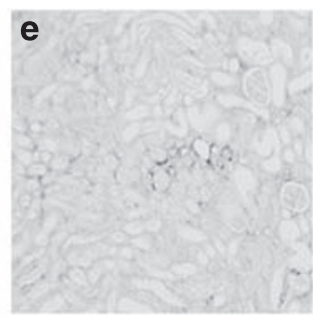

Fas+Imida treated

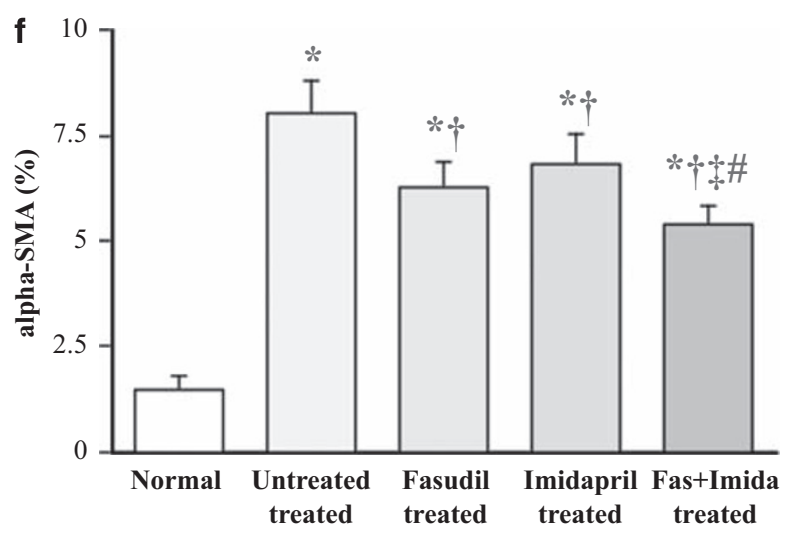

$* \mathrm{P}<0.05$ vs. untreated

$* * \mathrm{P}<0.01$ vs. untreated

$\uparrow \mathrm{P}<0.05$ vs. fasudil-treated

$\ddagger \mathrm{P}<0.05$ vs. imidapril-treated

Figure 2 The $\alpha$-SMA-positive cells in the interstitium in normal kidney in sham-operated mice (a) and in obstructed kidneys in untreated (b), fasudil-treated (c), imidapril-treated (d) and fasudil- and imidapril-treated mice (e) 11 days after the production of UUO are shown. The $\alpha$-SMA-positive areas in the interstitium are shown in (f). Data are expressed as mean \pm s.d. The number of mice was $6-8$ in each group. ${ }^{*} P<0.05$ vs. normal, ${ }^{\dagger} P<0.05$ vs. untreated, ${ }^{\ddagger} P<0.05$ vs. fasudil-treated, ${ }^{\#} P<0.05$ vs. imidapril-treated mice.

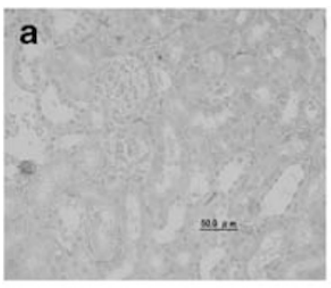

Normal

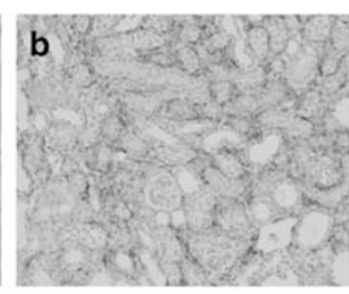

Untreated

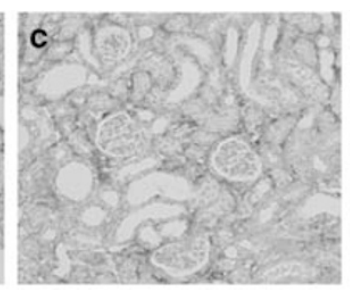

Fasudil

treated

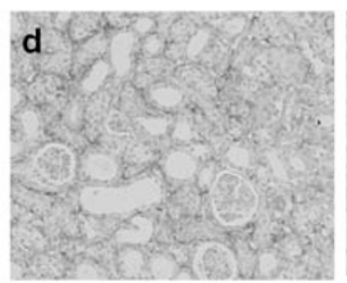

Imidapril

treated

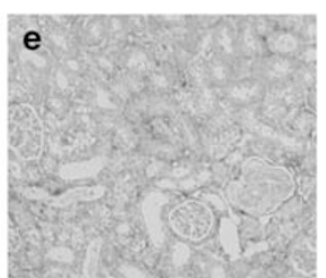

Fas+Imida treated

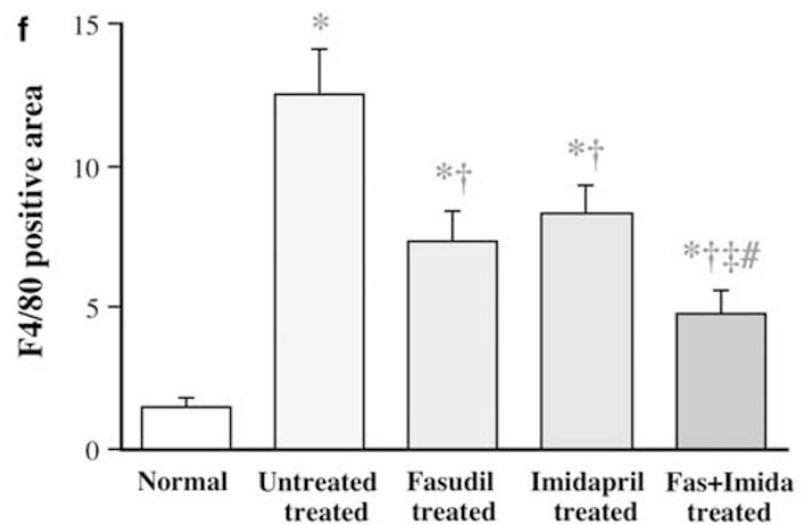

Figure 3 F4/80-positive cells in the interstitium in normal kidney in sham-operated mice (a) and in obstructed kidneys in untreated (b), fasudil-treated (c), imidapril-treated (d) and fasudil- and imidapril-treated mice (e) 11 days after the production of UUO are shown. The F4/80-positive areas in the interstitium are shown in (f). Data are expressed as mean \pm s.d. Number of mice was $6-8$ in each group. ${ }^{*} P<0.05$ vs. normal, ${ }^{\dagger} P<0.05$ vs. untreated, ${ }^{\ddagger} P<0.05$ vs. fasudil-treated, ${ }^{\#} P<0.05$ vs. imidapril-treated mice. 


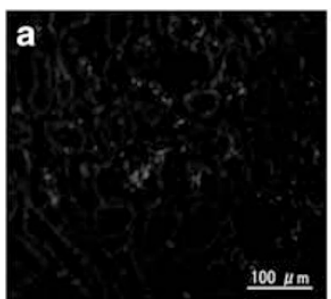

Normal

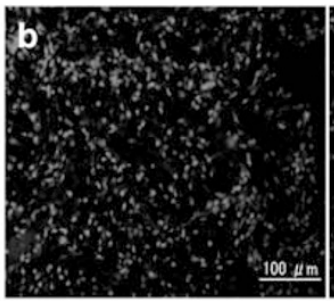

Untreated

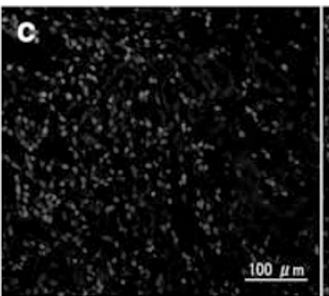

Fasudil treated

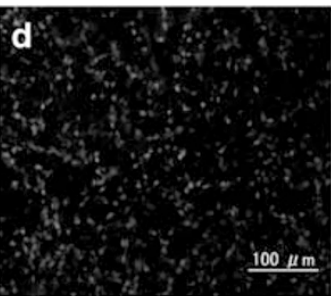

Imidapril treated

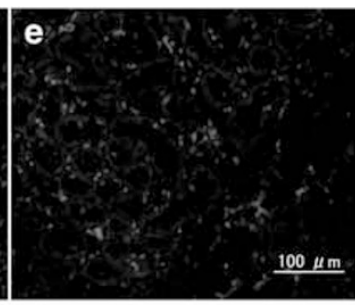

Fas +Imida

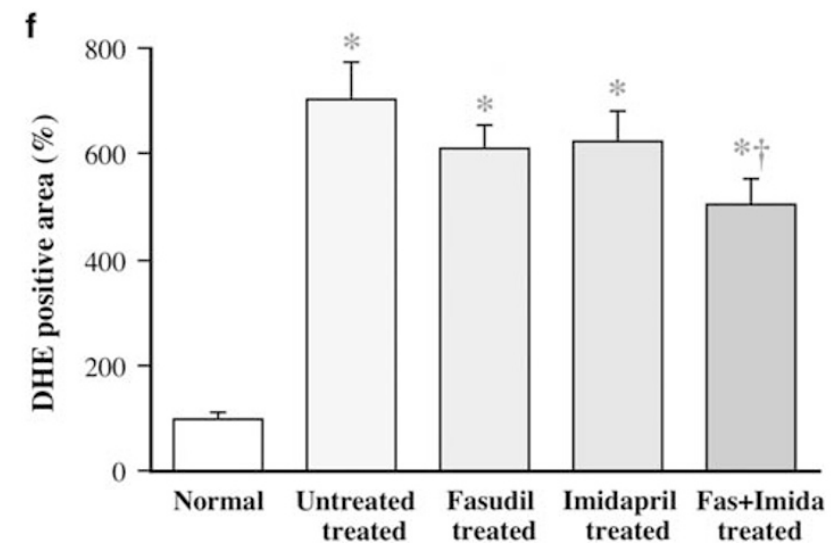

Figure 4 DHE staining was used to estimate superoxide generation. Representative DHE fluorescent photomicrographs in the interstitium in normal kidney in sham-operated mice (a) and in obstructed kidneys in untreated (b), fasudil-treated (c), imidapril-treated (d) and fasudil- and imidapril-treated mice (e) 11 days after the production of UUO are shown. The DHE-positive area in the interstitium is shown in (f) (normal kidney is shown in open bar, normalized as $100 \%$ ). Data are expressed as mean \pm s.d. The number of rats was $6-8$ in every group. ${ }^{*} P<0.05$ vs. normal, ${ }^{\dagger} P<0.05$ vs. untreated.

greater in the untreated-UUO mice compared with the sham-operated mice (Figures $4 \mathrm{a}$ and $\mathrm{b}$ ). Fasudil or imidapril treatment slightly reduced the fluorescence intensity in the interstitium (Figures $4 \mathrm{c}$ and $\mathrm{d}$ ), and the combination of fasudil and imidapril further reduced it (Figure 4e). The results of the quantitative analysis of fluorescence intensity in the obstructed kidney are shown in Figure $4 \mathrm{f}$. The mean fluorescence intensity values in the obstructed kidneys were markedly greater in the untreated-UUO mice than in the sham-operated mice $(P<0.01)$. Fasudil or imidapril treatment did not reduce the mean fluorescence intensity value; however, the reduction caused by the combination of fasudil and imidapril was significant $(P<0.05$ vs. untreated group) (Figure 4f).

\section{Renal gene expression levels in the five groups}

The mRNA levels of RhoA, RhoB and RhoC relative to the GAPDH mRNA levels in the renal cortex were significantly increased in obstructed kidney in UUO mice (about 1.5-2.5-fold) compared with those in the normal kidney. Long-term fasudil treatment did not change the mRNA levels of RhoA, RhoB or RhoC relative to the GAPDH mRNA levels (data not shown).

The mRNA expression levels of TGF- $\beta$, collagen-I and collagen-III, as assessed by real-time PCR at 11 days after the operation, are shown in Figures $5 \mathrm{a}-\mathrm{c}$. The mRNA expression levels of TGF- $\beta$ and collagen-I and the collagen-III/GAPDH ratio were markedly increased in the obstructed kidney in UUO mice (about 30-150-fold) compared with those in the normal kidney in the sham-operated mice $(P<0.01)$ (Figures $5 \mathrm{a}-\mathrm{c}$ ). Fasudil or imidapril monotreatment significantly reduced these increased mRNA expression levels compared with those in the untreated mice (both $P<0.05$ vs. untreated), and the combination of fasudil and imidapril further reduced them $(P<0.05$ vs. fasudil- or imidapril-treated group) (Figures $5 \mathrm{a}-\mathrm{c}$ ).

The mRNA expression levels of MCP-1 and TNF- $\alpha$, as assessed by real-time PCR at 11 days after the operation, are shown in Figures $5 \mathrm{~d}$ and e. The mRNA expression level of MCP- 1 and the TNF- $\alpha /$ GAPDH ratio were markedly increased in the obstructed kidney in the UUO mice (about 60-150-fold) compared with those in the normal kidney in the sham-operated mice $(P<0.01)$ (Figures $5 \mathrm{~d}$ and e). Fasudil or imidapril monotreatment significantly reduced the increased mRNA expression level of MCP-1/GAPDH $(P<0.05)$, but not the TNF- $\alpha$ / GAPDH ratio compared with those in the untreated mice. The combination of fasudil and imidapril further reduced the MCP-1/ GAPDH ratio $(P<0.05)$ and significantly reduced the TNF- $\alpha /$ GAPDH ratio (Figures $5 \mathrm{~d}$ and e).

The mRNA expression levels of NADPH subunits, as assessed by real-time PCR at 11 days after the operation, are shown in Figures $5 \mathrm{f}-\mathrm{j}$. The mRNA expression level of the NADPH subunits/ GAPDH ratio was increased in the obstructed kidney in the UUO mice (about 2.5-30-fold) compared with that in the normal kidney in the sham-operated mice $(P<0.01)$ (Figures $5 \mathrm{f}-\mathrm{j})$. Fasudil or imidapril monotreatment did not decrease the mRNA expression level of NADPH subunits/GAPDH ratio. However, the combination of fasudil and imidapril significantly decreased the p22phox/GAPDH ratio, gp91phox/GAPDH ratio, p40phox/GAPDH ratio, p47phox/GAPDH ratio and $\mathrm{p} 67 \mathrm{phox} / \mathrm{GAPDH}$ ratio (Figures $5 \mathrm{f}-\mathrm{j}$ ). 

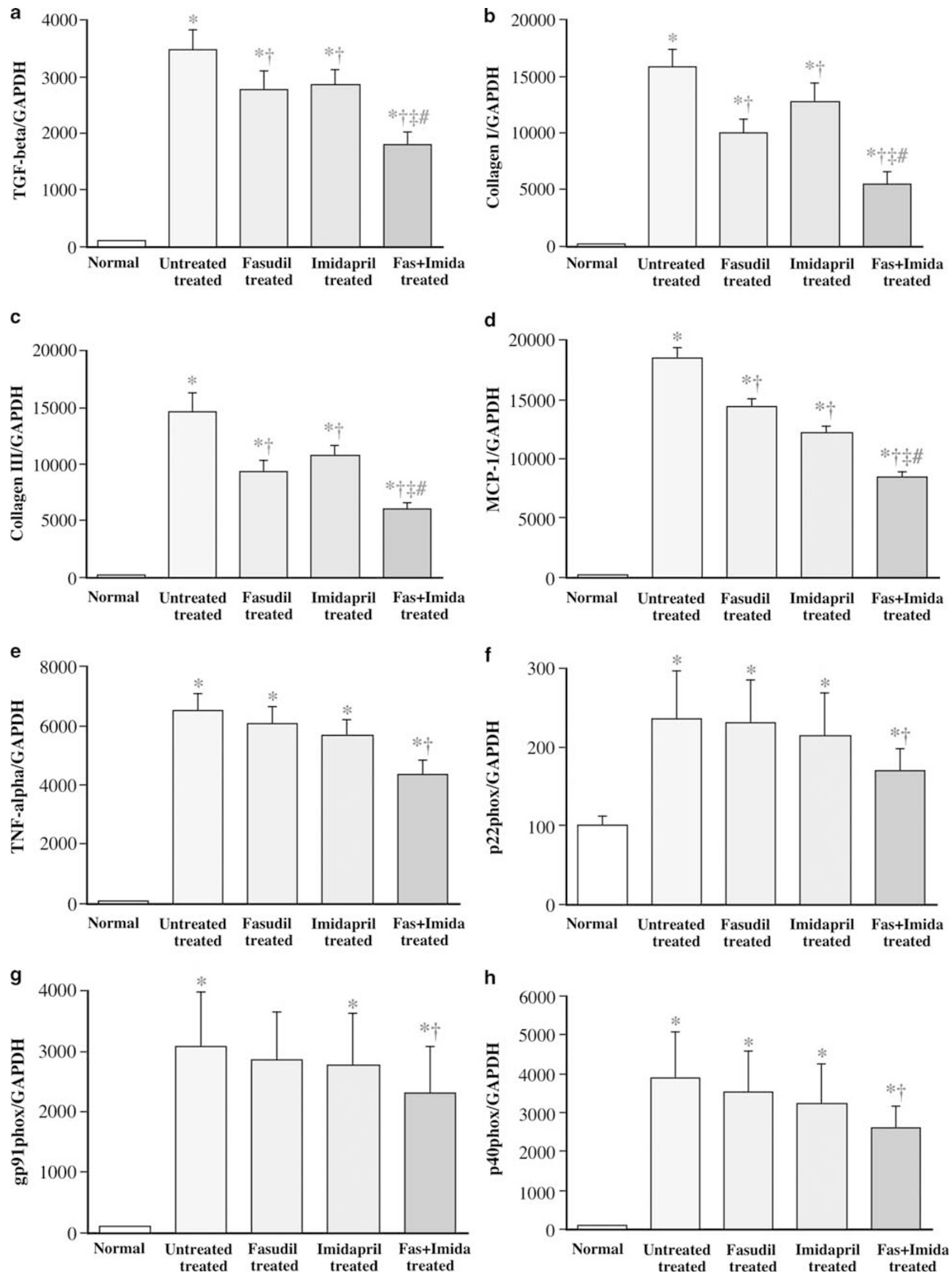

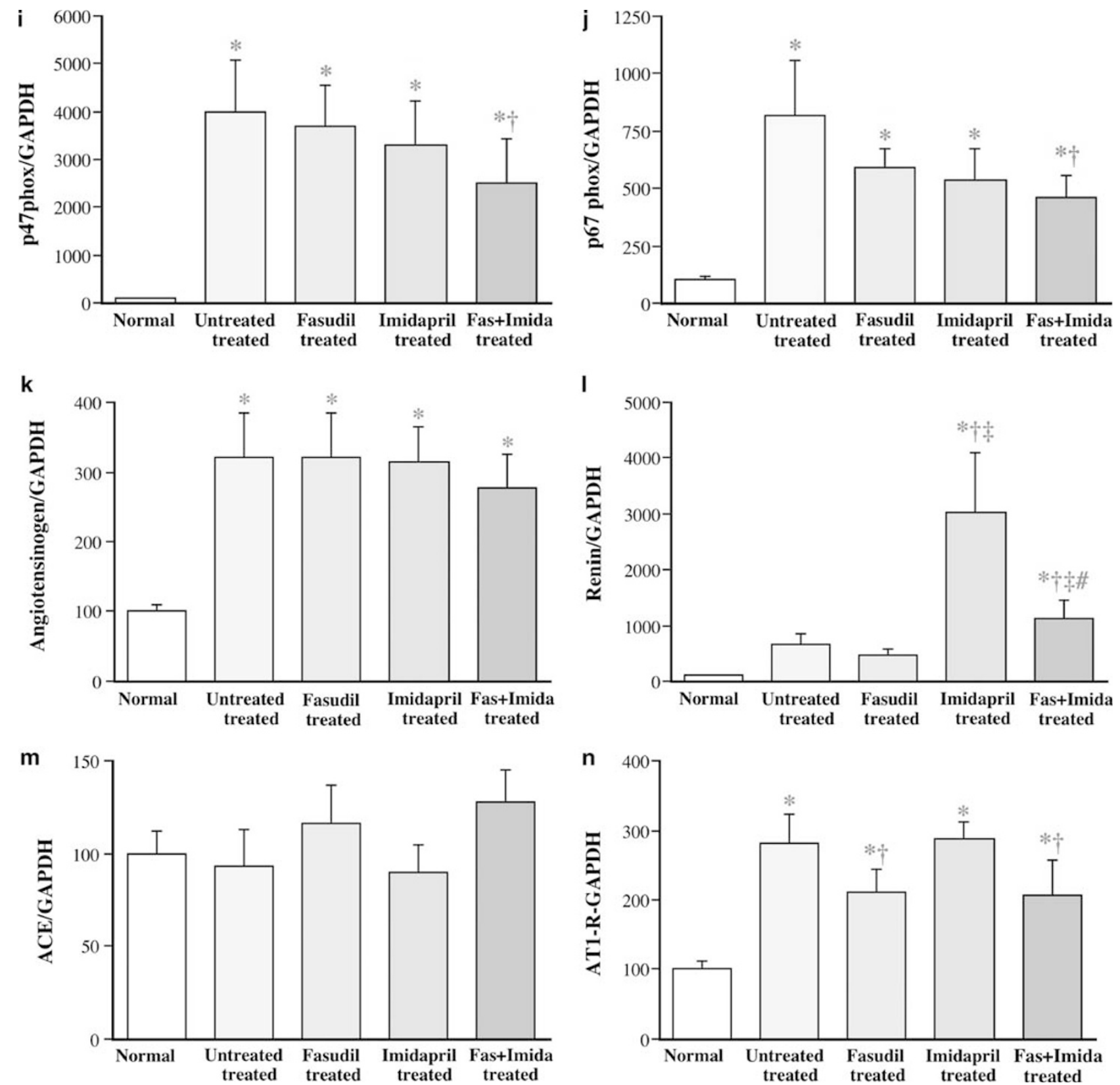

Figure 5 Continued.

Figure $5(\mathbf{a}-\mathbf{c})$ The mRNA expression levels of TGF- $\beta$ (a), collagen-I (b) and collagen-III (c) normalized to GAPDH mRNA levels in normal kidney in shamoperated mice and in obstructed kidneys in untreated, fasudil-treated, imidapril-treated and fasudil- and imidapril-treated mice 11 days after production of the UUO are shown (normal kidney is shown in open bar, normalized as 100\%). Data are expressed as mean \pm s.d. The number of rats was $6-8$ in every group. ${ }^{*} P<0.05$ vs. normal, ${ }^{\dagger} P<0.05$ vs. untreated, ${ }^{\ddagger} P<0.05$ vs. fasudil-treated, ${ }^{\#} P<0.05$ vs. imidapril-treated mice. (d, e) The mRNA expression levels of MCP-1 (d) and TNF- $\alpha$ (e) normalized to GAPDH mRNA levels in normal kidney in sham-operated mice and in obstructed kidneys in untreated, fasudil-treated, imidapril-treated and fasudil- and imidapril-treated mice 11 days after the production of UUO are shown (normal kidney is shown in open bar, normalized as $100 \%$ ). Data are expressed as mean \pm s.d. The number of rats was $6-8$ in every group. ${ }^{*} P<0.05$ vs. normal, ${ }^{\dagger} P<0.05$ vs. untreated, $\ddagger P<0.05$ vs. fasudiltreated, $\# P<0.05$ vs. imidapril-treated mice. (f-j) The mRNA expression levels of p22phox (f), gp91phox (g), p40phox (h), p47phox (i) and 67phox (h) normalized to GAPDH mRNA levels in normal kidney in sham-operated mice and in obstructed kidneys in untreated, fasudil-treated, imidapril-treated and fasudil- and imidapril-treated mice 11 days after production of UUO are shown (normal kidney is shown in open bar, normalized as 100\%). Data are expressed as mean \pm s.d. The number of rats was $6-8$ in every group. ${ }^{*} P<0.05$ vs. normal, ${ }^{\dagger} P<0.05$ vs. untreated, ${ }^{\ddagger} P<0.05$ vs. fasudil-treated, ${ }^{\#} P<0.05$ vs. imidapril-treated mice. $(\mathbf{k}-\mathbf{n})$ The mRNA expression levels of angiotensinogen $(\mathbf{k})$, renin (I), ACE $(\mathbf{m})$ and AT1-R (n) normalized to GAPDH mRNA levels in normal kidney in sham-operated mice and in obstructed kidneys in untreated, fasudil-treated, imidapril-treated and fasudil- and imidapril-treated mice 11 days after the production of UUO are shown (normal kidney is shown in open bar, normalized as 100\%). Data are expressed as mean \pm s.d. The number of rats was $6-8$ in every group. ${ }^{*} P<0.05$ vs. normal, ${ }^{\dagger} P<0.05$ vs. untreated, ${ }^{\ddagger} P<0.05$ vs. fasudil-treated, ${ }^{\sharp} P<0.05$ vs. imidapril-treated mice. 
The mRNA expression levels of ANG, renin, ACE and the AT1-R, as assessed by real-time PCR at 11 days after the operation, are shown in Figures $5 \mathrm{k}-\mathrm{n}$. The mRNA expression levels of ANG and the AT1-R/ GAPDH ratio were increased in obstructed kidney in the UUO mice (about threefold) compared with that in normal kidney in the shamoperated mice, but renin and the ACE/GAPDH ratio were not significantly increased (Figures $5 \mathrm{k}-\mathrm{n}$ ). Fasudil monotreatment decreased the mRNA expression level of the AT1-R/GAPDH ratio. In contrast, imidapril monotherapy significantly increased the mRNA expression levels of the renin/GAPDH ratio (Figures 51 and $n$ ). The combination of fasudil and imidapril significantly decreased the renin/GAPDH ratio compared with imidapril monotherapy; however, it was still higher than that in the untreated mice (Figure 5l). The combination of fasudil and imidapril decreased the mRNA expression AT1-R/GAPDH ratio as observed for fasudil monotreatment (Figure 5n).

\section{DISCUSSION}

This study provides the first experimental evidence that combination therapy involving a Rho-kinase inhibitor and an ACE inhibitor is more beneficial than either agent alone for treating renal fibrosis induced by UUO.

In this study, the mRNA expressions of RhoA, RhoB and RhoC were significantly increased in the obstructed kidney in UUO mice, suggesting that a Rho/Rho-kinase pathway is activated in this model. ${ }^{7}$ Long-term fasudil or imidapril treatment did not change these mRNA levels, which are consistent with the earlier reports. ${ }^{7,12}$

It has been well established that UUO causes renal fibrosis through many molecular mechanisms. ${ }^{21}$ Although renal fibrosis caused by chronic kidney disease is a clinically very important problem, only a few useful therapeutic drugs for renal fibrosis exist and its efficacy is limited. We and other investigators recently reported that Rho-kinase inhibitors attenuate glomerulosclerosis in salt-induced hypertensive rats, ${ }^{9}$ subtotally nephrectomized spontaneously hypertensive rats, ${ }^{10}$ severely hypertensive rats, ${ }^{12}$ aldosterone-induced glomerular injury in rats ${ }^{13}$ and diabetic renal injury in rats. ${ }^{14}$ Therefore, in this study, to investigate whether the combination of a Rho-kinase inhibitor and an ACE inhibitor is beneficial for treating renal fibrosis, we used a renal fibrosis model induced by UUO.

We showed that renal fibrosis induced by UUO caused an increase in myofibroblast differentiation, as shown by the $\alpha$-SMA-positive area, which causes excess deposition of the extracellular matrix and renal fibrosis. ${ }^{21}$ Interestingly, the combination therapy suppressed renal $\alpha$-SMA-positive area to a larger extent than each monotherapy, suggesting that the combination therapy may be more effective for reducing renal myofibroblast differentiation in this model. In addition, renal fibrosis, as assessed by Mason's trichrome staining, was increased in untreated-UUO mice. Given that collagen accumulation is responsible for renal fibrosis, ${ }^{22}$ the renal fibrosis in UUO can be explained, at least in part, by the enhancing of the TGF- $\beta /$ collagen pathway. ${ }^{23}$ A great deal of investigation has been conducted to understand the cellular and molecular mechanisms of renal fibrosis in this model. ${ }^{24}$ At present, one of the most important molecular mechanism of renal fibrosis in this model is increase of TGF- $\beta .^{23-25}$ Earlier studies have shown that macrophage/monocyte infiltration occurred in the kidney $4 \mathrm{~h}$ after acute ureteral obstruction and its peak response occurred at $24 \mathrm{~h}$ after UUO and stabilized thereafter. ${ }^{26}$ Another study also showed a highly significant correlation between the increasing number of interstitial macrophage/monocyte and the cortical TGF- $\beta$ mRNA levels. ${ }^{27}$ Taken together, these results suggest that macrophage/monocyte is one potential source for the increased gene expression of TGF- $\beta$. In this study, F4/80-positive cells in the interstitium was significantly decreased in fasudil and combination group. Therefore, one possible mechanism of reduction of TGF- $\beta$ mRNA levels induced by fasudil or combination may be due to the reduction of macrophage/monocyte infiltration.

Recent studies have shown that an important mechanism underlying the development of tubulointerstitial fibrosis is epithelial-tomesenchymal transition. ${ }^{28,29}$ Epithelial-to-mesenchymal transition, a process by which differentiated epithelial cells undergo a phenotypic conversion that gives rise to the matrix-producing fibroblasts and myofibroblasts, is increasingly recognized as an integral part of tissue fibrogenesis after injury. Emerging evidence suggests that TGF- $\beta$ initiates the transition of renal tubular epithelial cells to myofibroblasts. Thus, fasudil or combination therapy is more effective at reducing renal fibrosis than monotherapy because of inhibition of macrophage/monocyte infiltration, leading to inhibition of the TGF$\beta /$ collagen gene expression, epithelial-to-mesenchymal transition and myofibroblast differentiation pathway.

Cellular reactive oxygen species are reported to be increased in renal disease, and they have been shown to contribute to the pathogenesis of chronic kidney disease..$^{30,31}$ Oxidative stress is known to enhance the TGF- $\beta$ /collagen cascade. ${ }^{32}$ In this study, the mRNA expression of NADPH oxidase subunits such as p47phox, p40phox, p67phox, p22phox and gp91phox was increased in the UUO kidney compared with the sham-operated kidney. ${ }^{33}$ Consistent with these findings, superoxide anion generation in the renal interstitium was increased as evaluated by DHE staining. Rho-kinase inhibitor or ACE inhibitor monotherapy did not attenuate the DHE-positive area or the mRNA expression levels of NADPH oxidase subunits. Only the combination of Rho-kinase inhibitor and ACE inhibitor significantly decreased the DHE-positive area. This reduction of the DHE-positive area appeared to be due to the change in the synthesis of superoxide by NADPH oxidase because the mRNA expression levels of NADPH oxidase subunits were only significantly reduced by the combination of the Rho-kinase inhibitor and the ACE inhibitor.

Earlier studies showed that macrophage/monocyte infiltration has a critical function in the development of renal interstitial fibrosis in a UUO model. ${ }^{21}$ MCP-1 mRNA expression is closely associated with macrophage/monocyte infiltration. ${ }^{7}$ Therefore, in this study, we measured macrophage/monocyte infiltration through the F4/80positive area using immunohistochemistry techniques. We found that F4/80-positive area significantly increased in UUO model with the increase of MCP-1 mRNA levels. Importantly, this increase in F4/80positive area was significantly attenuated by fasudil or imidapril monotherapy and was further reduced by the combination of fasudil and imidapril treatment. These findings, together with the fact that macrophage/monocyte infiltration can stimulate the oxidative stress, the TGF- $\beta /$ collagen cascade and cytokine production, ${ }^{34}$ support the notion that the reduction of renal macrophage/monocyte infiltration induced by the combination therapy is involved in the amelioration of renal fibrosis.

As shown above, fasudil and imidapril have similar actions on TGF- $\beta /$ collagen cascade, myofibroblast differentiation and oxidative stress generation. However, the effect of these drugs on renal tissue RAS was different. Imidapril increased the expression of renin mRNA probably through a negative feedback mechanism; however, it did not change the level of ANG, ACE or AT1-R mRNA, which is consistent with the results of an earlier report. ${ }^{35}$ Fasudil did not affect the expression of ANG, renin or ACE mRNA, but slightly decreased that of AT1-R mRNA. We earlier reported that fasudil improved renal impairment in a salt-induced hypertensive rat model without changing plasma renin 
activity. ${ }^{9}$ Thus, regarding the effect of Rho-kinase inhibitors on the tissue RAS level, further study is required.

We have a limitation in this study. Although we have shown that mRNA level of Rho-kinase was increased, we did not measure tissue Rho-kinase activity in this study. However, we earlier showed that increased mRNA levels of Rho-A, B and C was associated with phosphorylated MYPT level, a marker of Rho-kinase activity. ${ }^{15}$ Collectively, these results suggest that Rho-kinase tissue activity was increased in this model. We did not measure ACE activity in this study, either. However, the BP reduction and increased mRNA levels of renin in ACEI treatment group strongly suggest that tissue ACE activity was deduced to be increased in UUO model.

Renal interstitial fibrosis is a progressive and potentially lethal disease caused by diverse clinical entities including urinary tract obstruction, chronic inflammation and diabetes. ${ }^{36}$ However, there are few renoprotective drugs at present that have been proved by large clinical trial. In addition, their effects are limited. Therefore, a new drug or combination therapy including ACE inhibitors is desired. In this study, we examined the effect of a combination of an ACE inhibitor and a Rho-kinase inhibitor on renal fibrosis induced by UUO and found that combination therapy is more effective than either monotherapy. These results suggest that the combination of an ACE inhibitor and a Rho-kinase inhibitor may be a new therapeutic approach in the renal interstitial fibrosis in the future.

In conclusion, we first showed that the combination of a Rhokinase inhibitor and an ACE inhibitor improved renal interstitial fibrosis more than monotherapy and that the effects of the combination therapy were associated with the inhibition of myofibroblast differentiation, the TGF- $\beta$ /collagen cascade, oxidative stress and cytokine production.

\section{CONFLICT OF INTEREST}

The authors declare no conflict of interest.

\section{ACKNOWLEDGEMENTS}

This work was supported, in part, by Scientific Research Grants-in-Aid 18590787 and 20590837 from the Ministry of Education, Culture, Sports, Science, and Technology of Japan; by the Science Research Promotion Fund from the Promotion and Mutual Aid Corporation for Private Schools of Japan; by a Research Grant for Cardiovascular Diseases (19C-7) from the Ministry of Health, Labour, and Welfare and by the Seki Minato Prize. We thank Mr Hisato Hirata, Mr Yoshifumi Machida, Ms Masako Minato, Ms Keiko Ishikawa, Ms Machiko Sakata, Ms Fumie Yokotsuka and Ms Noriko Suzuki for providing technical assistance.

1 Takai Y, Sasaki T, Matozaki T. Small GTP-binding proteins. Physiol Rev 2001; 81: 153-208.

2 Fukata Y, Amano M, Kaibuchi K. Rho-Rho-kinase pathway in smooth muscle contraction and cytoskeletal reorganization of non-muscle cells. Trends Pharmacol Sci 2001; 22: 32-39.

3 Uehata M, Ishizaki T, Satoh H, Ono T, Kawahara T, Morishita T, Tamakawa H, Yamagami K, Inui J, Maekawa M, Narumiya S. Calcium sensitization of smooth muscle mediated by a Rho-associated protein kinase in hypertension. Nature 1997; 389: 990-994.

4 Shimokawa H, Hiramori K, linuma H, Hosoda S, Kishida H, Osada H, Katagiri T, Yamauchi K, Yui Y, Minamino T, Nakashima M, Kato K. Anti-anginal effect of fasudil, a Rho-kinase inhibitor, in patients with stable effort angina: a multicenter study. J Cardiovasc Pharmacol 2002; 40: 751-761.

5 Shimokawa H, Takeshita A. Rho-kinase is an important therapeutic target in cardiovascular medicine. Arterioscler Thromb Vasc Biol 2005; 25: 1767-1775.

6 Nishikimi T, Matsuoka H. Molecular mechanisms and therapeutic strategies of chronic renal injury: renoprotective effect of rho-kinase inhibitor in hypertensive glomerulosclerosis. J Pharmacol Sci 2006; 100: 22-28.

7 Nagatoya K, Moriyama T, Kawada N, Takeji M, Oseto S, Murozono T, Ando A, Imai E, Hori M. Y-27632 prevents tubulointerstitial fibrosis in mouse kidneys with unilateral ureteral obstruction. Kidney Int 2002; 61: 1684-1695.
8 Satoh S, Yamaguchi T, Hitomi A, Sato N, Shiraiwa K, Ikegaki I, Asano T, Shimokawa H. Fasudil attenuates interstitial fibrosis in rat kidneys with unilateral ureteral obstruction. Eur J Pharmacol 2002; 455: 169-174.

9 Nishikimi T, Akimoto K, Wang X, Mori Y, Tadokoro K, Ishikawa Y, Shimokawa H, Ono H, Matsuoka H. Fasudil, a Rho-kinase inhibitor, attenuates glomerulosclerosis in Dahl saltsensitive rats. J Hypertens 2004; 22: 1787-1796.

10 Kanda T, Wakino S, Hayashi K, Homma K, Ozawa Y, Saruta T. Effect of fasudil on Rhokinase and nephropathy in subtotally nephrectomized spontaneously hypertensive rats. Kidney Int 2003; 64: 2009-2019.

11 Ishikawa Y, Nishikimi T, Akimoto K, Ishimura K, Ono H, Matsuoka H. Long-term administration of rho-kinase inhibitor ameliorates renal damage in malignant hypertensive rats. Hypertension 2006; 47: 1075-1083.

12 Nishikimi T, Koshikawa S, Ishikawa Y, Akimoto K, Inaba C, Ishimura K, Ono H, Matsuoka $\mathrm{H}$. Inhibition of Rho-kinase attenuates nephrosclerosis and improves survival in salt-loaded spontaneously hypertensive stroke-prone rats. J Hypertens 2007; 25: 1053-1063.

13 Sun GP, Kohno M, Guo P, Nagai Y, Miyata K, Fan YY, Kimura S, Kiyomoto H, Ohmori K, Li DT, Abe Y, Nishiyama A. Involvements of Rho-kinase and TGF-beta pathways in aldosterone-induced renal injury. J Am Soc Nephrol 2006; 17: 2193-2201.

14 Peng F, Wu D, Gao B, Ingram AJ, Zhang B, Chorneyko K, McKenzie R, Krepinsky JC. RhoA/Rho-kinase contribute to the pathogenesis of diabetic renal disease. Diabetes 2008; 57: 1683-1692.

15 Koshikawa S, Nishikimi T, Inaba C, Akimoto K, Matsuoka H. Fasudil, a Rho-kinase inhibitor, reverses L-NAME exacerbated severe nephrosclerosis in spontaneously hypertensive rats. J Hypertens 2008; 26: 1837-1848.

16 Ono H, Saitoh M, Ono Y, Ishimitu T, Matsuoka H. Imidapril improves L-NAMEexacerbated nephrosclerosis with TGF-beta 1 inhibition in spontaneously hypertensive rats. J Hypertens 2004; 22: 1389-1395.

17 Cravedi P, Remuzzi G. Treating the kidney to cure the heart. Kidney Int Supp/ 2008; 111: S2-S3.

18 Nishikimi T, Inaba-lemura C, Ishimura K, Tadokoro K, Koshikawa S, Ishikawa K, Akimoto K, Hattori Y, Kasai K, Minamino N, Maeda N, Matsuoka H. Peptide/natriuretic peptide receptor-A (NPR-A) system has inhibitory effects in renal fibrosis in mice. Regul Pept 2009; 154: 44-53.

19 Higashi M, Shimokawa H, Hattori T, Hiroki J, Mukai Y, Morikawa K, Ichiki T, Takahashi $\mathrm{S}$, Takeshita A. Long-term inhibition of Rho-kinase suppresses angiotensin II-induced cardiovascular hypertrophy in rats in vivo: effect on endothelial $\mathrm{NAD}(\mathrm{P}) \mathrm{H}$ oxidase system. Circ Res 2003; 93: 767-775.

20 Nishikimi T, Hagaman JR, Takahashi N, Kim HS, Matsuoka H, Smithies O, Maeda N. Susceptibility to heart failure in response to volume overload in mice lacking natriuretic peptide receptor-A gene. Cardiovasc Res 2005; 66: 94-103.

21 Chevalier RL, Forbes MS, Thornhill BA. Ureteral obstruction as a model of renal interstitial fibrosis and obstructive nephropathy. Kidney Int 2009; 75: 1145-1152.

22 Wada T, Sakai N, Matsushima K, Kaneko S. Fibrocytes: a new insight into kidney fibrosis. Kidney Int 2007; 72: 269-273.

23 Bascands JL, Schanstra JP. Obstructive nephropathy: insights from genetically engineered animals. Kidney Int 2005; 68: 925-937.

24 Chevalier RL, Forbes MS, Thornhill BA. Ureteral obstruction as a model of renal interstitial fibrosis and obstructive nephropathy. Kidney Int 2009; 75: $1145-1152$.

25 Sato M, Muragaki Y, Saika S, Roberts AB, Ooshima A. Targeted disruption of TGFbeta1/Smad3 signaling protects against renal tubulointerstitial fibrosis induced by unilateral ureteral obstruction. J Clin Invest 2003; 112: 1486-1494.

26 Schreiner GF, Harris KP, Purkerson ML, Klahr S. Immunological aspects of acute ureteral obstruction: immune cell infiltrate in the kidney. Kidney Int 1988; 34: 487-493.

27 Diamond JR, Kees-Folts D, Ding G, Frye JE, Restrepo NC. Macrophages, monocyte chemoattractant peptide-1, and TGF-beta 1 in experimental hydronephrosis. Am J Physiol 1994; 266: F926-F933.

28 Hills CE, Squires PE. TGF-beta1-induced epithelial-to-mesenchymal transition and therapeutic intervention in diabetic nephropathy. Am J Nephrol 2010; 31: 68-74.

29 Zeisberg M, Hanai J, Sugimoto H, Mammoto T, Charytan D, Strutz F, Kalluri R. BMP-7 counteracts TGF-betal-induced epithelial-to-mesenchymal transition and reverses chronic renal injury. Nat Med 2003; 9: 964-968.

30 Cachofeiro V, Goicochea M, de Vinuesa SG, Oubia P, Lahera V, Luo J. Oxidative stress and inflammation, a link between chronic kidney disease and cardiovascular disease. Kidney Int Suppl 2008; 111: S4-S9.

31 Tojo A, Asaba K, Onozato ML. Suppressing renal NADPH oxidase to treat diabetic nephropathy. Expert Opin Ther Targets 2007; 11: 1011-1018.

32 Paravicini TM, Touyz RM. NADPH oxidases, reactive oxygen species, and hypertension: clinical implications and therapeutic possibilities. Diabetes Care 2008; 31(Suppl 2): S170-S180.

33 Sugiyama H, Kobayashi M, Wang DH, Sunami R, Maeshima Y, Yamasaki Y, Masuoka N, Kira S, Makino $\mathrm{H}$. Telmisartan inhibits both oxidative stress and renal fibrosis after unilateral ureteral obstruction in acatalasemic mice. Nephrol Dial Transplant 2005; 20: 2670-2680.

34 Tojo A, Asaba K, Onozato ML. Suppressing renal NADPH oxidase to treat diabetic nephropathy. Expert Opin Ther Targets 2007; 11: 1011-1018.

35 Ferrario CM, Jessup J, Gallagher PE, Averill DB, Brosnihan KB, Ann Tallant E, Smith $\mathrm{RD}$, Chappell MC. Effects of renin-angiotensin system blockade on renal angiotensin(1-7) forming enzymes and receptors. Kidney Int 2005; 68: 2189-2196.

36 Remuzzi G, Bertani T. Pathophysiology of progressive nephropathies. N Eng/ J Med 1998; 339: 1448-1456. 